\title{
Digitale politische Partizipation - Spannungsfeld zwischen MyPolitics und OurPolitics
}

\author{
Andreas Ladner $\cdot$ Andreas Meier
}

Eingegangen: 19. September 2014 / Angenommen: 9. Oktober 2014 / Online publiziert: 17. Oktober 2014

(C) Springer Fachmedien Wiesbaden 2014

Zusammenfassung Der Beitrag untersucht soziale und politische Aspekte digitaler politischer Partizipation. Einerseits müssen die Optionen für MyPolitics ausgeschöpft werden, indem Instrumente für elektronische Abstimmungen und Wahlen bereitgestellt werden. Andererseits sollte das Potenzial politischer Partizipation genutzt und Plattformen für OurPolitics gefördert werden. Solche Plattformen, ergänzt durch geeignete Matching-Verfahren, bringen Interessierte mit ähnlichen Anliegen zusammen und fördern politische Gestaltungskraft. Sie sind ein erster Schritt zum Public Memory einer digitalen Gesellschaft und bereichern nachfolgende Generationen mit ihrem Gedankengut.

Schlüsselwörter Demokratie 3.0 - Digitale Gesellschaft · Partizipation · Wahlhilfesysteme $\cdot$ Bürgerbeteiligung $\cdot$ Elektronische Abstimmungen und Wahlen · Public Memory

\section{Netzgeneration}

Ihr macht euch lustig über unsere Sozialen Netzwerke und unseren neuen Gebrauch des Wortes ,Freund'. Habt ihr es je vermocht, euch in Gruppen zusammenzufinden, die von so beträchtlichem Umfang sind, daß die Zahl ihrer Mitglieder sich denjenigen der Menschen nähert? Und ist es nicht klug, sich den anderen zunächst virtuell zu nähern, um sie nicht zu verletzen? Ihr habt nur Angst vor den

\footnotetext{
A. Ladner $(\bowtie)$

IDHEAP, Quartier UNIL Mouline, Universität Lausanne,

1015 Lausanne, Schweiz

E-Mail: andreas.ladner@unil.ch
}
A. Meier
Departement für Informatik, Universität Fribourg,
Boulevard de Pérolles 90,
1700 Fribourg, Schweiz 
neuen politischen Formen, die aus diesen Unternehmungen hervorgehen und die alten, obsolet gewordenen wegfegen könnten (Serres 2013, S. 57 und 58).

Die zitierte Aussage stammt nicht von einem digitalen Eingeborenen (digital native), der mit dem Internet aufgewachsen ist und sich rund um die Uhr in sozialen Netzen tummelt. Sie stammt auch nicht von einem Vertreter der Piratenpartei, der sich für die Stärkung der Bürgerrechte, mehr direkte Demokratie und Informationsfreiheit stark macht. Nein, das Zitat stammt von einem digitalen Einwanderer (digital immigrant), der bei der Geburtsstunde des Web bereits neunundfünfzig Jahre alt war und an der Sorbonne in Paris und an der Stanford University in Kalifornien Philosophie und Wissenschaftsgeschichte lehrt.

Als Mitglied der Netzgeneration spielt das Alter keine Rolle. Wichtiger ist, dass sich die Nutzer über örtliche und zeitliche Grenzen hinweg begegnen. Das Web dient ihnen nicht nur beim elektronischen Austausch von Information oder bei der rechnergestützten Arbeitsbewältigung, es fördert die Bildung von Gemeinschaften. Der Cyberspace wächst zu einem Raum, in dem sich dessen Bewohner treffen, Beziehungen und Wissen austauschen, gemeinsame Projekte vorantreiben und Sprachgrenzen und kulturelle Barrieren überwinden.

Für eDemocracy und eGovernment werden Infrastrukturen entwickelt, Austausch- und Wissensplattformen bereitgestellt und unterschiedliche Serviceangebote gemacht (Meier 2009a). Verhaltensregeln und Sicherheitsmechanismen dienen dazu, die eigene Privatsphäre zu schützen und Missbräuche abwehren zu können.

Die Netzgeneration formt digitale Partizipationsoptionen und pocht darauf, das demokratische Zusammenleben neu zu erfinden. Dabei ergeben sich zwei sich ergänzende Optionen, die eine geht von den persönlichen, individuellen Partizipationsrechten aus, die andere von kollektiven und deliberativen Teilnahmemöglichkeiten:

MyPolitics: Bürgerinnen und Bürger, die regelmässig eVoting (elektronische Abstimmungen) und eElection (elektronische Wahlen) betreiben, legen sich eine Plattform unter MyPolitics an. Sie speichern darauf ihre politischen Präferenzen, Überlegungen zu Wahlen und Abstimmungen sowie ihre Wahlresultate. Eventuell öffnen sie Teile ihres politischen Tagebuches gegenüber einzelnen Familienmitgliedern, Freunden oder Mitgliedern unterschiedlicher politischer Gruppierungen und es enstehen Political Communities of Interest.

OurPolitics: Möchte man sich stärker für einzelne politische Themen engagieren, geht man einen Schritt weiter und wird Mitglied der gemeinnützigen Plattform OurPolitics. Dazu hinterlegt man ein politisches Profil, das jederzeit abgeändert oder ergänzt werden kann. Mit Machting-Verfahren werden einem Landkarten zu ausgewählten politischen Anliegen generiert, wo man Bürger oder Politiker mit ähnlichen Präferenzen treffen und Austausch pflegen kann. Eventuell entstehen Political Communities of Practice, d. h. vernetzte Gruppenmitglieder, die gemeinsam politische Projekte entwickeln und verfolgen, in die sie Zeit und Wissen investieren.

Beide Arten von Gemeinschaften, Political Community of Interest wie Political Community of Practice, lassen sich mit Informations- und Kommunikationstechno- 
logien unterstützen. Im Folgenden sollen zuerst elektronische Wahlen und Abstimmungen thematisiert resp. Optionen für MyPolitics aufgezeigt werden, bevor die webbasierte politische Partizipation OurPolitics am Beispiel von Wahlhilfesystemen und Erweiterungen aufgezeigt wird.

\section{Webbasierte Abstimmungs- und Wahlsysteme}

\subsection{Bausteine von MyPolitics}

Mit der Nutzung von Webtechnologien können Bürgerinnen und Bürger ihre politischen Rechte (Stimmabgabe, Wahl, Unterschriften bei Referenden und Initiativen etc.) elektronisch wahrnehmen (Meier 2009b). Die Abb. 1 zeigt die Vielfalt bei elektronischen Abstimmungen und Wahlen.

Bei Web Polls steht die webbasierte und unverbindliche Umfrage zu einem öffentlichen Sachgeschäft oder einem politischen Programm im Vordergrund. Danach können elektronisch ermittelte Resultate diskutiert und entsprechende Initiativen weiterentwickelt werden. Hier müssen die Bürgerinnen und Bürger nicht in jedem Fall eindeutig identifiziert und mit digitalen Signaturen (siehe Public Key Infrastructure, z. B. in Meier 2009a, S. 97 ff.) auf ihre Authentifikation hin überprüft werden. Vielmehr wird ein Stimmungsbild zu einem öffentlichen Sachgeschäft oder Thema eingefangen.

Elektronische Abstimmungssysteme lassen sich an Versammlungsorten oder in geschützten Räumlichkeiten installieren. Jeder Teilnehmer verfügt über ein elektro-

\begin{tabular}{|c|c|c|c|}
\hline & Definition & Merkmale & Beispiele \\
\hline Web Polls & $\begin{array}{l}\text { unverbindliche } \\
\text { Abstimmung oder Wahl }\end{array}$ & $\begin{array}{l}\text { - keine Garantie auf } \\
\text { Korrektheit } \\
\text { - keine Garantie auf } \\
\text { Anonymität }\end{array}$ & $\begin{array}{l}\text { unverbindliche } \\
\text { Bürgerbefragung }\end{array}$ \\
\hline $\begin{array}{l}\text { elektronische } \\
\text { Abstimmungs- } \\
\text { Systeme }\end{array}$ & $\begin{array}{l}\text { elektronische } \\
\text { Abstimmungsgeräte } \\
\text { in einem Saal }\end{array}$ & $\begin{array}{l}\text { - automatische Erfassung } \\
\text { - unmittelbare Ergebnis- } \\
\text { anzeige }\end{array}$ & $\begin{array}{l}\text { Abstimmung } \\
\text { im Parlament }\end{array}$ \\
\hline $\begin{array}{l}\text { elektronische } \\
\text { Wahlmaschinen }\end{array}$ & $\begin{array}{l}\text { elektronische } \\
\text { Abstimmungsgeräte } \\
\text { und Urnen im Wahllokal }\end{array}$ & $\begin{array}{l}\text { - Stimmberechtigung der } \\
\text { Wähler manuell prüfen } \\
\text { - Ergebnisse einzelner } \\
\text { Maschinen manuell } \\
\text { zusammenfassen }\end{array}$ & $\begin{array}{l}\text { Wahlen in } \\
\text { Belgien }\end{array}$ \\
\hline $\begin{array}{l}\text { vernetzte } \\
\text { Wahlmaschinen }\end{array}$ & $\begin{array}{l}\text { vernetzte Abstimmungs- } \\
\text { geräte in öffentlichen } \\
\text { Wahllokalen }\end{array}$ & $\begin{array}{l}\text { - Ergebnisse automatisch } \\
\text { für alle angeschlossenen } \\
\text { Maschinen ermittelbar }\end{array}$ & $\begin{array}{l}\text { Lokalwahlen in } \\
\text { England }\end{array}$ \\
\hline $\begin{array}{l}\text { eVoting und } \\
\text { eElection }\end{array}$ & $\begin{array}{l}\text { orts- und zeitunabhängige } \\
\text { Abstimmungen u. Wahlen } \\
\text { durch Geräte (Handy, } \\
\text { Palmtop, digitales TV u.a.) }\end{array}$ & $\begin{array}{l}\text { - Registrierung eindeutig } \\
\text { - Abstimmung oder Wahl } \\
\text { bleibt geheim }\end{array}$ & $\begin{array}{l}\text { Abstimmungen in } \\
\text { Genf und Zürich }\end{array}$ \\
\hline
\end{tabular}

Abb. 1 Vielfalt elektronischer Abstimmungen und Wahlen nach Meier (2009b, S. 52) 
nisches Abstimmungs- oder Wahlgerät. Nach der Stimmabgabe werden die einzelnen Stimmen elektronisch zusammengezählt und auf Anzeigetafeln veröffentlicht. Solche Abstimmungssysteme sind meistens in Parlamentssälen fest installiert. Zudem lassen sie sich mit begrenztem Aufwand bei öffentlichen Versammlungen ad hoc aufbauen und nutzen.

Als weitere Option können Wahlcomputer oder elektronische Wahlmaschinen in den Wahllokalen aufgestellt und vernetzt werden. Wahlcomputer sind Geräte, die mit einer Abstimmungs- und Wahlsoftware versehen werden. Elektronischen Wahlmaschinen sind Spezialgeräte, die ausschliesslich für elektronische Abstimmungen und Wahlen konzipiert sind. Damit soll verhindert werden, dass die Abgabe von Stimmen und das Auslesen der Resultate manipuliert werden kann.

Webbasierte Abstimmungen zu einem Sachgeschäft (eVoting) und Wahlen von Mandatsträgern (eElection) können in einem definierten Zeitfenster orts- und zeitunabhängig durchgeführt werden. Die Identifikation und Authentifikation der Wähler erfolgt z. B. mit der Hilfe einer Public Key Infrastructure oder durch geeignete Sicherheitssysteme, um eine Manipulation der Abstimmungs- und Wahlresultate auszuschliessen.

Elektronische Abstimmungen und Wahlen unterscheiden sich in den Vor- und Nachbearbeitungsphasen gegenüber herkömmlichen Abstimmungs- und Wahlverfahren (vgl. Abb. 2), falls die Vorteile elektronischer Austauschbeziehungen ausgeschöpft werden. Durch erweiterte Informations- und Diskussionsangebote (vgl. Prozessschritte eDiscussion und ePosting) erhofft man sich, Bürgerinnen und Bürger vermehrt für politische Anliegen zu gewinnen und die Bildung von Gemeinschaften zu fördern.

In Abb. 2 werden die folgenden Prozessschritte unterschieden:

eDiscussion: Im Vorfeld von Abstimmungen und Wahlen lässt sich der Meinungsbildungsprozess fördern, in dem neben Informationsangeboten auch Diskussionsforen aufgeschaltet werden. Damit können die Teilnehmer Einschätzungen und Bewertungen einholen. Zusätzliche Abonnementsdienste ermöglichen, Entscheidungsgrundlagen zu erhalten sowie Änderungen bei Sachthemen zu erfahren. Wich-

Abb. 2 eVoting und eElection als Teil einer Prozesskette nach (Meier 2009a, S. 169)
- elektronischer

Stimmzettel

- Einschätzungen

fakultativ

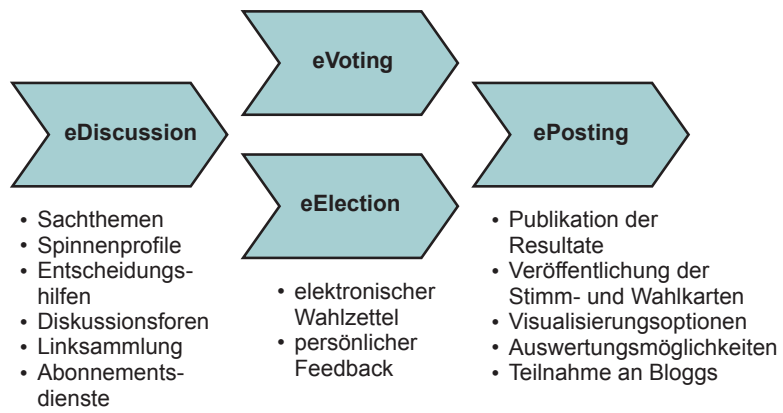


tige Hintergrundinformationen, Beiträge aus Diskussionsforen, Entscheidungshilfen etc. werden bei Bedarf auf MyPolitics abgelegt.

eVoting: Jeder Teilnehmer einer elektronischen Abstimmung muss sich bei der Behörde identifizieren und registrieren lassen, um einen elektronischen Wahlzettel zu erhalten. Die elektronische Abstimmung erfolgt anonym. Bei Bedarf kann die Behörde neben dem Stimmzettel eine fakultative Erhebung über die vorliegenden Themen beifügen, um z. B. bei Vorgehens- und Umsetzungsfragen einen Feedback von den Bürgerinnen und Bürgern zu erhalten. Ein Abbild des elektronischen Wahlzettels, ev. ergänzt durch das persönliche Feedback, wird auf MyPolitics gespeichert.

eElection: Die politischen Präferenzen und Programme der zu wählenden Kandidaten werden im Prozessschritt eDiscussion publiziert. Damit fällt es dem Wählenden einfacher, den elektronischen Wahlzettel auszufüllen. Auch hier muss sich der Wähler registrieren, um von der Behörde einen gültigen Wahlzettel zu erhalten. Eventuell drängt es sich auf, fakultative Zusatzfragen durch die Bürgerinnen und Bürger beantworten zu lassen. Wahlzettel wie beantwortete Zusatzfragen werden fakultativ auf MyPolitics abgelegt.

ePosting: Die Resultate von elektronischen Abstimmungen und Wahlen werden auf dem eGovernment-Portal der Behörde publiziert; je nach Wunsch werden Teile auf MyPolitics übertragen. Öffentlichkeit sowie Presse können diese studieren, kommentieren und weiterverwenden. Neben den Bürgerinnen und Bürgern des jeweiligen Landes können beliebige Anspruchsgruppen die Resultate einsehen. Ein erster Schritt für ein Public Memory ist damit gemacht (vgl. Abschn. 4).

Mit geeigneten Visualisierungs- und Auswertungswerkzeuge bis hin zu der Verwendung von Empfehlungssystemen (vgl. Abschn. 3) lassen sich politische Angebote sowie Kandidaten analysieren und besprechen. Public Blogs ermöglichen, die elektronische Abstimmung oder Wahl über den Stimm- und Wahltag hinaus zu kommentieren und zu vertiefen.

\subsection{Fallbeispiel eVoting in der Schweiz}

Wenn man vergleicht, wie stark webbasierte Anwendungen in praktisch sämtlichen Lebensbereichen zum Einsatz kommen, so erstaunt es, dass dem eVoting (teilweise werden auch die Begriffe I-Voting oder Remote Internet Voting verwendet) der Durchbruch noch nicht gelungen ist. Abgesehen von Estland, das als Pionierland des elektronischen Wählens gilt, gibt es kaum Länder, die die Urne durch Internetapplikationen zu ersetzen beginnen.

In der Schweiz wurde das Thema elektronisch Abstimmen (eVoting) und Wählen (eElection) zu Beginn der Nullerjahre auf Bundesebene aufgenommen. Der Bundesrat liess 2002 verlauten, dass die Schweiz nicht nur bei der direkten, sondern auch bei der elektronischen Demokratie eine weltweit führende Rolle einnehmen soll. ,Mit der Einführung des Vote électronique könnte die Schweiz auf dem Gebiet der elektronischen Demokratie eine Vorreiterrolle im internationalen Bereich wahrnehmen. Ihr 
praktisches Beispiel könnte zeigen, dass die neuen elektronischen Möglichkeiten in grossräumigeren staatlichen Gebilden, insbesondere in supranationalen Organisationen, die Einführung von Elementen der direkten Demokratie erleichtern und fördern könnten' (Bundesrat 2002).

Nach einer ersten Etappe mit Pilotversuchen in den drei Kantonen Genf, Neuenburg und Zürich, die in enger Zusammenarbeit mit dem Bund durchgeführt und von ihm auch finanziell unterstützt wurden, entschloss sich der Bundesrat 2006 für eine schrittweise und risikobewusste Einführung des Vote électronique. Darauf folgte eine erweiterte Versuchsphase, die 2008 mit dem Inkrafttreten der Gesetzes- und Verordnungsänderungen zu den politischen Rechten begann. Die neuen Normen sahen zur Hauptsache vor, dass sich weitere Kantone an den Versuchen beteiligten konnten und dass die Bewilligung nach fünf pannenfreien Versuchen erweitert werden konnte. Für die Zeit 2007 bis 2011 blieb die Zahl der elektronisch Abstimmenden auf 10\% der Wählerschaft beschränkt. Bei obligatorischen Referenden, bei denen auch das Ständemehr entscheidend ist, sollten zusätzlich nicht mehr als $20 \%$ der betroffenen kantonalen Elektorate über den Vote électronique abstimmen können. Zudem wurden die Voraussetzungen für einen funktionstüchtigen Einbezug der Auslandschweizerinnen und Auslandschweizer in Versuche mit dem Vote électronique geschaffen.

Am 13. Dezember 2013 wurde der Bundesrat etwas vorsichtiger und revidierte die Bestimmungen für die Durchführung von Versuchen mit der elektronischen Stimmabgabe. Die neuen Rechtsgrundlagen definieren - gestützt auf den dritten Bericht des Bundesrats zu Vote électronique vom Juni 2013- die Bedingungen für die Ausdehnung des elektronischen Stimmkanals. Erst nach Umsetzung der erhöhten Sicherheitsanforderungen kann nun die Anzahl Stimmberechtigte, die an den Versuchen teilnehmen darf, erhöht werden. Damit reagierte der Bundesrat auf Sicherheitslücken, auf die beim Genfer System in der Öffentlichkeit hingewiesen wurden. Das Bewilligungsverfahren für die Versuche wurde hingegen vereinfacht und die Bundeskanzlei wurde ermächtigt, zusätzliche Ausführungsbestimmungen rund um die elektronische Stimmabgabe zu erlassen.

Auch wenn das Ganze insgesamt nun etwas gemächlicher vonstatten geht als ursprünglich geplant, so ist das Projekt nach wie vor unterwegs. Bei den nationalen Wahlen 2011 konnten die 22.000 Auslandschweizerinnen und Auslandschweizer der Kantone Basel-Stadt, St. Gallen, Graubünden und Aargau erstmals elektronisch wählen. Es handelte sich dabei um den ersten Versuch mit Wahlen auf Bundesebene. Damit wurde ein wichtiges Etappenziel erreicht. Die Bundeskanzlei hat sich nun zum Ziel gesetzt, 2015 eine Mehrheit der Auslandschweizer Stimmberechtigten elektronisch an den Wahlen auf Bundesebene teilnehmen zu lassen.

Anlässlich der eidgenössischen Volksabstimmung vom 9. Februar 2014 führten bereits zwölf Kantone Versuche mit der elektronischen Stimmabgabe durch, mehrheitlich mit Auslandschweizern. Auch diese Versuche erfüllten die Anforderungen des Bundes und gelten als erfolgreich.

Auf der Ebene der Kantone schreiten die Projekte ebenfalls voran. Nachdem der Kanton Zürich zwischenzeitlich sein Projekt gestoppt hatte, ist er 2013 dem 2009 gegründeten überkantonalen ,Consortium Vote électronique', welches vom Kanton Aargau geleitet wird, beigetreten. Auch hier sollen zuerst die Auslandschweizer elek- 
tronisch an den Wahlen teilnehmen, danach soll diese Möglichkeit auf alle Stimmberechtigten ausgeweitet werden.

\subsection{Ausblick - Fuzzy Voting}

Lotfi A. Zadeh hat 1965 mit seinem Forschungspapier ,Fuzzy Sets` den Grundstein zur unscharfen Logik (Fuzzy Logic) gesetzt (vgl. Zadeh 1965). Unscharfe Mengen sind Mengen, bei welchen man die Zugehörigkeit der Elemente zur Menge mit einer Zugehörigkeitsfunktion misst. Die Werte der Zugehörigkeit können beliebige Werte auf dem Einheitsintervall annehmen. Der Wert 1 entspricht dem Wert wahr, 0 drückt eine falsche Aussage aus und 0.33 z. B. bedeutet, dass die Aussage zu einem Drittel richtig scheint.

Die klassische Logik basiert auf einer zweielementigen Menge, d. h. 1 steht für wahr und 0 für falsch. Die Welt des Diskurs ist eine Schwarzweisslandschaft. Bei der unscharfen Logik hingegen sind Grautöne erlaubt. Sogar das ganze Spektrum wird zugelassen, d. h. es gibt unendlich viele Abstufungen zwischen weiss und schwarz. Diese differenzierte Sicht der Dinge war lange Zeit umstritten, bis man in der Steuerungstechnik damit erste Erfolge erzielte. Vor allem Japan spielte eine Vorreiterrolle, indem das MITI (Ministry of International Trade and Industry) mit über vierzig Firmen ein Labor für die Anwendung der unscharfen Logik in der Technik gründete. Unscharfe Mengen sind nicht nur bei der Steuerung technischer Geräte nützlich, sondern lassen sich im betriebs- und volkswirtschaftlichen Umfeld anwenden.

Unscharfe Ansätze können für die Stimmabgabe selbst verwendet werden (CorteReal 2007). Dies bedeutet, dass der Wähler neben Ja (Wert 1) oder Nein (Wert 0) einen differenzierteren Grauwert (Wert zwischen 0 und 1) einlegen darf. Eine Stimmabgabe mit dem Wert 0.6 würde dann interpretiert, dass der Wähler mit $60 \%$ eine Vorlage unterstützt und zu $40 \%$ ablehnt.

Die Abb. 3 zeigt ein mögliches Szenario bei herkömmlichen Stimmen (crisp votes; Ja oder Nein) und unscharfen Stimmen (fuzzy votes; Ja oder Nein oder, sowohl als auch'). Nehmen wir an, es liegen zwei Konzepte vor, mit den Bezeichnungen Black und White. Im klassischen Fall müssen die Wähler sich für Black oder White entscheiden und entsprechend ihre volle Stimme für das gewählte Konzept einlegen. Im unscharfen Fall können die Wähler beiden Konzepten je einen Teil ihrer Stimme

Abb. 3 Fiktives Beispiel für Fuzzy Voting

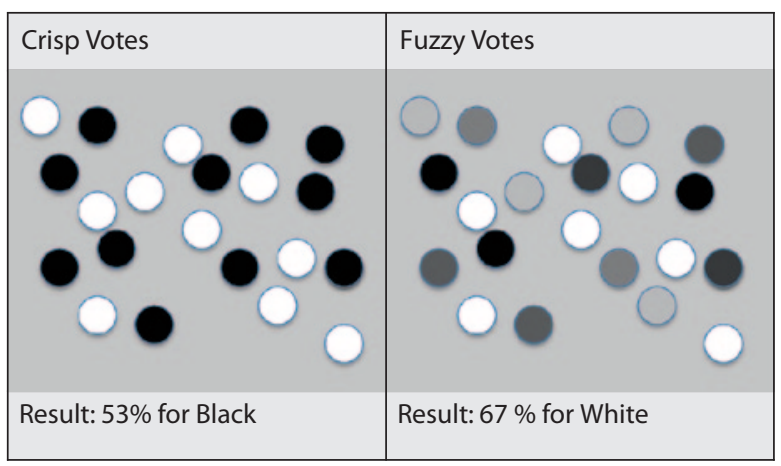


vergeben (z. B. $70 \%$ für White und $30 \%$ für Black) oder die ganze Stimme auf ein Konzept setzen (z. B. 100\% für White). Dies kann bei knappen Stimmverhältnissen dazu führen, dass im klassischen Fall das Konzept Black gutgeheissen wird (hier mit $53 \%$ ). Würde hingegen unscharf abgestimmt, hätte das Konzept White mit $67 \%$ die Nase vorn.

\section{Webbasierte Wahlhilfesysteme}

Voting Advice Applications (VAAs) sind Webplattformen, welche den Wählenden aufzeigen, welche Partei ihren politischen Positionen am nächsten steht. Sie verbinden MyPolitics mit OurPolitics und fördern webbasierte Gemeinschaften. Solche Plattformen bringen Wählende und Kandidaten/Parteien zusammen und schaffen Political Communities of Interest. Zudem stützen sie den Wahlakt und die Erwartung, dass politische Ideen und Projekte in Political Communities of Practice realisiert werden, aber dazu später.

\subsection{Bausteine von OurPolitics}

Herzstück von VAAs ist eine Datenbank, in der die zur Wahl stehenden politischen Parteien und Kandidierenden mit ihrem politischen Profil erfasst sind. Erstellt wird dieses Profil aufgrund ihrer Einstellungen und Positionen zu politischen Themen und Sachfragen. Diese können erfragt, aufgrund von Dokumenten (Parteiprogrammen, Wahlplattformen) bestimmt oder von Experten eingeschätzt werden.

Die Wählenden respektive die Webnutzer erstellen bei Bedarf ihr persönliches politisches Profil. Dazu nehmen sie zu denselben politischen Fragen Stellung wie die politischen Kandidaten. Der Computer berechnet danach die Übereinstimmung zwischen ihrem Profil und den Profilen der Parteien oder Kandidierenden und zeigt an, wie gross die Gemeinsamkeiten sind. So erfährt der Webnutzer, welche Parteien und welche Kandidierenden ihm politisch am nächsten stehen. Zudem werden ihnen in der Regel noch weitere Informationen über die Parteien oder die Kandidaten angeboten. Sie können nachschauen, wie diese bei den einzelnen Political Issues positioniert sind, finden Links zu deren Webseiten oder Angaben, wie sie mit ihnen in Kontakt treten können. Wie die Webnutzer mit dieser Information und vor allem mit den Übereinstimmungswerten oder der in einem räumlichen Modell dargestellten Nähe zu Parteien oder Kandidaten umgehen, ist ihnen selbst überlassen. Impliziert ist jedoch die Vorstellung, dass sich die Wählenden in der Regel von Parteien und Kandidierenden vertreten lassen möchten, die ihnen politisch möglichst ähnlich sind und deshalb dieser Wahlempfehlung folgen. Diese Form des Wählens wird in der politikwissenschaftlichen Literatur als Issue Voting bezeichnet.

Als Pionierland für solche Plattformen gelten die Niederlande, wo den Wählerinnen und Wählern mit dem ,Stemwijzer' bereits 1989 eine erste Wahlhilfe - allerdings als Papierversion - angeboten wurde. Auf die Print-Version folgte 1998 die erste Online-Version. Mittlerweilen gibt es in den Niederlanden auch andere Wahlhilfen, wie z. B. ,Kieskompas‘. Der, Wahl-O-Mat' aus Deutschland hingegen gibt keine Wahlempfehlungen, sondern lediglich Informationen über anstehende Wahlen, wie 
z. B. zur Wahl des sächsischen Landtags am 31. August 2014. Die ,Wahlkabine“ ist ein Projekt in Österreich, die ,Cabina Elettorale“ ein italienisches (vgl. De Rosa 2010). In Grossbritannien heisst die Plattform ,Who-Do-I-Vote-For' und der ,Doe De Stemtest' ist ein Produkt aus Belgien. Weitere solche Plattformen gibt oder gab es in Finnland, Luxemburg, Portugal, sowie auch in Bulgarien, Kanada, Türkei oder im Irak. Auch wenn diese Angebote nicht in allen Ländern dieselbe Institutionalisierung erfahren haben und zu einer festen Begleiterscheinung der Wahlen geworden sind, wie dies etwa in den Niederlanden, Deutschland und der Schweiz der Fall ist, werden sie heute von Millionen von Wählenden genutzt.

\subsection{Fallbeispiel SmartVote ${ }^{1}$ in der Schweiz}

Die bekannteste Wahlhilfe-Plattform in der Schweiz ist www.smartvote.ch, die seit 2003 existiert. Im Wahljahr 2011 wurde neben SmartVote mit www.vimentis.ch eine ähnliche, aber deutlich weniger bekannte Webseite angeboten. SmartVote ist ganz besonders auf das relativ komplizierte Schweizer Wahlsystem zugeschnitten, bei dem sich die Wählenden nicht nur für Parteien sondern auch für die einzelnen Kandidierenden entscheiden können. So standen im Kanton Zürich bei den Nationalratswahlen 2011 beispielsweise 802 Kandidaten auf 30 Listen zur Auswahl. Die Wählenden hatten die Möglichkeit, eine Liste von 34 Namen mit Personen aus unterschiedlichen Parteien zusammenzustellen. Sie konnten aber auch eine vorgedruckte Parteiliste nehmen, sie einfach so einwerfen oder sie zuerst noch abändern, indem sie darauf Kandidierende aus anderen Parteien aufführten (Panaschieren). Auch konnten Kandidierende doppelt aufgeführt werden (Kumulieren). Wollen Wählende all diese Wahlmöglichkeiten vollständig ausschöpfen, benötigen sie weitreichende Informationen, nicht nur über die Parteien, sondern auch über die Kandidierenden. SmartVote kann neben Parteiprofilen auch Profile für jeden einzelnen Kandidaten erstellen. Ohne Computerunterstützung wäre eine solche Informationsfülle kaum zu verarbeiten.

SmartVote erfreut sich in der Schweiz einer grossen Popularität. Bei den Nationalratswahlen 2007 wurden nahezu eine Million Wahlempfehlungen ausgestellt (Ladner et al. 2008), was rund 20\% der Wahlberechtigten entsprechen würde. Bei diesen Zahlen gilt es allerdings zu berücksichtigten, dass sich manche Leute mehrere Wahlempfehlungen ausstellen lassen. In wissenschaftlichen Studien wird von rund 300.000 Benutzenden ausgegangen (Ladner 2009). Bei einer Wahlbeteiligung von 2.37 Mio. (48.3\% der Wahlberechtigten) wären das etwas mehr als zwölf Prozent der Wählenden oder knapp sechs Prozent der Wahlberechtigten. Eigene Berechnungen mit dem Datensatz für die Wahlen 2007 bestätigen diese Hochrechnungen, und weitere Auswertungen der Daten für die Wahlen 2011 zeigen, dass die Benutzung auf mindestens $15 \%$ der Wählenden angewachsen ist.

Gründe für die zunehmende Popularität dieser Online-Wahlhilfen sind sicher zuerst einmal in der wachsenden Bedeutung und Verbreitung des Internets zu finden. In praktisch sämtlichen Lebensbereichen holt man sich Rat im Internet, da liegt es nahe, dass man sich dort auch über die zu wählenden Parteien und Kandidaten informiert. Deutlich gewachsen ist zudem der Bekanntheitsgrad der Wahlhilfen selbst. Die

${ }^{1}$ Für weitere Angaben über SmartVote vgl. auch Ladner 2009, 2012. 
Medien gehen Partnerschaften mit den Anbietern dieser Plattformen ein. Häufig werden die Tools direkt in die Online-Plattformen der Medien integriert, um den Lesern so einen direkten Zugang zu ermöglichen. Auch die Berichterstattung über VAAs ist markant angestiegen. Dies hat mitunter damit zu tun, dass viele dieser Plattformen attraktive Visualisierungsformen anbieten, mit denen die unterschiedlichen politischen Positionierungen von Parteien und Kandidaten verdeutlicht werden können.

Ein weiterer Grund für die wachsende Bedeutung dieser Tools ist wohl auch, dass sie dem veränderten Wahlverhalten entgegen kommen. Gemäss den Erkenntnissen der Wahlforschung reduzieren die Wählenden die Komplexität der Politik mit Hilfe von Cues and Shortcuts (Dalton und Wattenberg 1993, S. 193). Als alte und traditionelle Fingerzeige und Abkürzungen galten in früheren Jahren die Zugehörigkeit zu einer sozialen Gruppe, wie zum Beispiel Arbeiterschaft oder Katholiken, und die - häufig über die Familien vermittelte Nähe zu politischen Parteien. Seit den 1970erJahren haben die bis dazumal relativ stabilen sozialen Cleavages an Bedeutung verloren (Franklin et al. 1992), was zu einem individuellerem Wahlverhalten geführt hat, welches sich stärker an politischen Präferenzen, am Leistungsausweis und am Erscheinungsbild von Kandidaten und Parteien orientiert (Dalton 1996, S. 346). Mit dem Auflösen der Parteibindungen steigt der Anteil der potentiellen Wechselwählenden. Die neuen Wahlplattformen nehmen diese Entwicklung direkt auf. Sie basieren auf der Idee, dass die Wählenden keine festen vorgefassten Parteibindungen haben, sondern bereit sind, ihren Wahlentscheid von einer systematischen Überprüfung der Übereinstimmung ihrer politischen Präferenzen mit denjenigen der zu Wahl stehenden Parteien und Kandidierenden abhängig zu machen.

\subsection{Ausblick - SmartParticipation}

SmartVote basiert auf einem relativ einfachen Algorithmus zur Ermittlung der politischen Nähe zwischen den Wählenden und den Kandidaten und auch ein Wahlentscheid wird in der Regel als einfache Entweder-Oder-Entscheidung aufgefasst. Denkbar sind auch alternative Modelle, die die Präferenzen besser berücksichtigen.

In einem Forschungsprojekt der Universität Fribourg ist das Wahlhilfesystem SmartVote zu einer Plattform SmartParticipation erweitert worden, in dem unscharfe Klassifikationsalgorithmen angewendet werden (Meier und Teran 2012, Teran 2014). Unschärfe bedeutet, dass die Kandidaten nicht scharf ihren politischen Parteien zugeordnet werden, sondern dass sie aufgrund ihrer Profile unterschiedliche Distanzen zu den Zentren verschiedener Parteien aufweisen. Damit kann ein Wähler nicht nur seine Position in der politischen Landschaft lokalisieren, sondern die Kandidaten mit ähnlichem Profil herauslesen (vgl. Abb. 4). Die Kandidaten, die nahe beim Wähler liegen, können unterschiedlichen politischen Parteien angehören.

Die Kandidaten, die sich einer politischen Wahl stellen, werden aufgrund eines ausgefüllten Fragebogens im mehrdimensionalen Raum dargestellt und mit einem unscharfen Clusterverfahren (fuzzy c-mean algorithm) gruppiert. Als Anzahl der zu bildenden Cluster gilt die Anzahl politischer Parteien. Unscharf bedeutet, dass einzelne Politikerinnen oder Politiker in unterschiedlichen Klassen vorkommen können, je nach ihrer Zugehörigkeit zu den Zentren unterschiedlicher Parteien. Zudem 


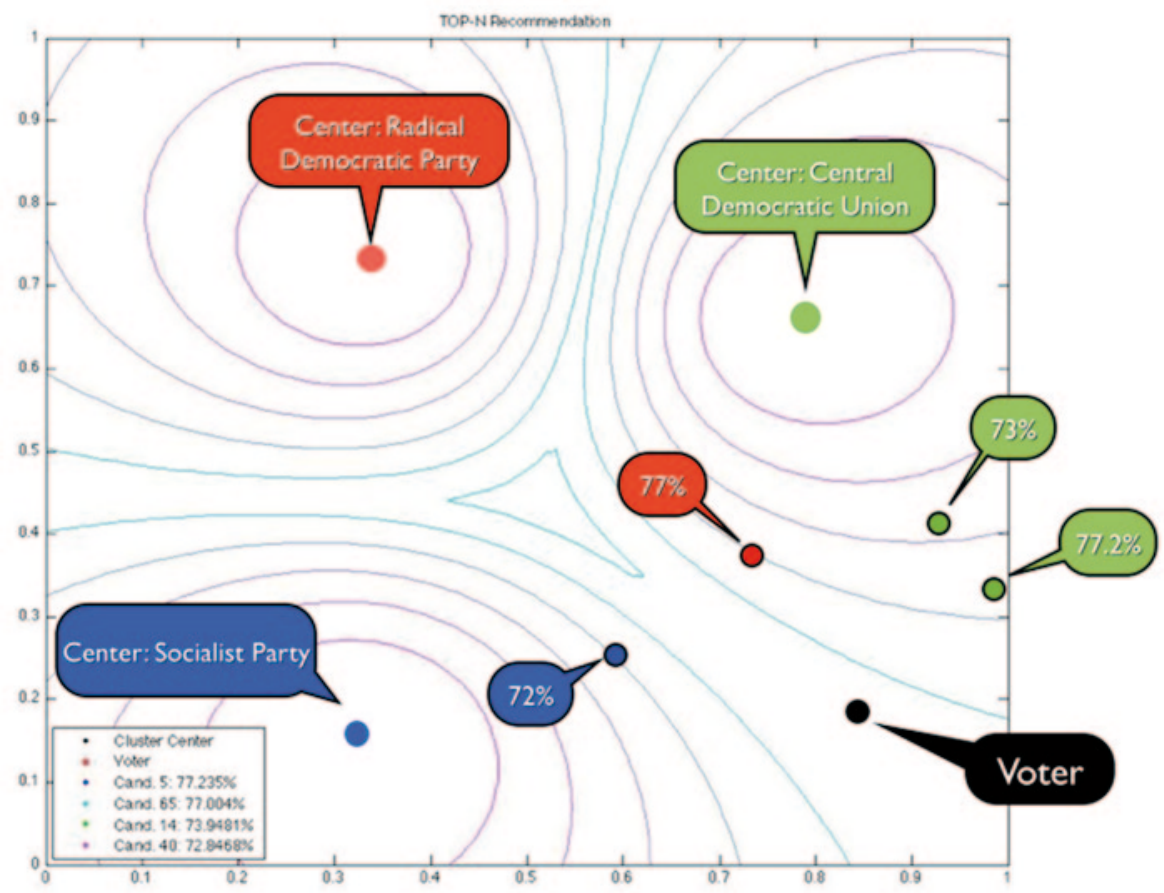

Abb. 4 Anzeigen der am nächsten gelegenen Wahlkandidaten nach (Teran 2014)

kann ein Wähler ebenfalls ein Profil ausfüllen und sich in der politischen Landkarte positionieren.

Das unscharfe Matching-Verfahren gibt dem Webnutzer somit alle Kandidaten, unabhängig ihrer Parteienzugehörigkeit, die in seiner Nähe liegen. Es basiert auf dem Vergleich der jeweiligen politischen Präferenzen. Dazu muss gesagt werden, dass der Abgleich politischer Präferenzen weiche Parameter wie Einstellungen der Kandidierenden, Charaktereigenschaften, Vertrauenswürdigkeit, Geradlinigkeit etc. nicht berücksichtigt. Mit anderen Worten wird die Wahl eines Kandidaten nicht nur aufgrund von Empfehlungssystemen politischer Präferenzen erfolgen, sondern auch Erfahrungswerte, Hinweise aus dem Freundeskreis, subjektive Einschätzungen etc. miteinbeziehen.

Natürlich können unscharfe Stimmen auch für die Wahl von Mandatsträgern verwendet werden. Bei einer klassischen Wahl gibt der Wähler seine Stimme zu 100 \% einem einzigen Kandidaten. Bei einem unscharfen Angebot verteilt der Wähler seine $100 \%$ an diejenigen Mandatsträger, die er teilweise unterstützen möchte. Anhänger der unscharfen Logik argumentieren damit, dass das menschliche Denken in den meisten Fälle unscharf ist und man nicht gezwungen werden sollte, die Welt in schwarz und weiss einzuteilen (Corte-Real 2007).

Die Plattform eParticipation ermöglicht zudem, den mehrdimensionalen Raum politischer Themen oder Teilthemen zu durchforsten. Mit geeigneten Such- und Zoomfunktionen können einzelne Themen oder Themenbündel analysiert werden. 
Gleichzeitig kann jederzeit abgefragt werden, welche Bürgerinnen und Bürger resp. Politiker sich in der Nähe befinden und entsprechend für das politische Anliegen sich engagieren wollen. Ein wichtiger Schritt zur Community Bildung (Political Community of Interest oder Practice) ist damit getan (vgl. Jeitziner und Meier 2010).

\section{Wege zum Public Memory}

Die Möglichkeiten - aber auch die Risiken - welche die interaktiven Partizipationsmöglichkeiten mit sich bringen, lassen sich erkennen, wenn wir gedanklich das elektronische Auswählen (eDiscussion, ePosting, VAAs) mit dem elektronischen Wählen (eVoting, eElection) verknüpfen. Dies führt uns abschliessend zu den beiden Stichworten Politcontrolling und Public Memory.

Die elektronischen Wahlhilfe-Webseiten machen es möglich - wie wir gesehen haben - auf eine informierte, oder zumindest informationsbasierte Art und Weise Entscheidungen zu treffen (Ladner et al. 2010). Dieser Prozess kann mit interaktiven Elementen (sei dies zwischen den Wählenden oder zwischen den Wählenden und den Kandidierenden) ergänzt werden. Die politische Nähe lässt sich mit Ansätzen unterschiedlicher Schärfe und Gewichtungen ermitteln. Das elektronische Wählen bietet demgegenüber die Möglichkeit, das Ergebnis der Auswahlprozesse auf einfache Weise zu übermitteln. Was liegt aus Sicht der Wählenden näher, als nach der Auswahl mit ein paar Klicks direkt zur Wahl zu schreiten? So werden schliesslich auch webbasiert Partner/innen gesucht oder Ferien gebucht.

Die webbasierte Entscheidungsfindung (Auswahl der Kandidaten und Parteien, die man wählen möchte) gewinnt an Bedeutung, je komplizierter das Selektionsproblem (Angebot, Wahlsystem) ist. Hat man, wie in der Schweiz, die Möglichkeit, zwischen Kandidaten unterschiedlicher Parteien auszuwählen (Panaschieren) und gewisse Kandidaten stärker zu gewichten (Kumulieren), und gilt es - wie vor allem in den grösseren Kantonen - eine grosse Zahl von Sitzen zu besetzen (34 im Kanton Zürich), so steigert sich der Nutzen dieser Webseiten beträchtlich. Besonders attraktiv ist die Möglichkeit, eine Wahlliste elektronisch zu erstellen. Auf diese kann man die von SmartVote empfohlenen Kandidaten übertragen und durch weitere Kandidaten ergänzen. Natürlich kann man unerwünschte Empfehlungen weglassen und gewisse Personen doppelt aufführen. Eine solche custom-made Wahlliste möchte man dann nicht von Hand abschreiben oder nochmals auf der offiziellen Wahlseite des Vote électronique eingeben müssen, sondern man will sie direkt übermitteln (Ladner 2012). Ist dies möglich, so wird die Zahl der Benutzer des elektronischen Wählens ansteigen, und wahrscheinlich hätte dies auch positive Auswirkungen auf die Wahlbeteiligung. Schon jetzt zeigen Untersuchungen, dass sich vor allem jüngere Personen von SmartVote zum Wählen animieren lassen (Ladner und Pianzola 2010).

Wenn man schon eine Webseite hat, auf der man sich über das Wahlangebot informiert und über die man auch wählen kann, so stellt sich ganz grundsätzlich die Frage, ob man über diese Webseite nicht noch mehr machen kann. Die Idee wäre, dass man sich eine persönliche, passwortgeschützte Webseite mit dem Namen MyPolitics erstellen lässt. Das politische Profil, welches man sich für die Matching-Prozedur bei SmartVote erstellen liess, wird gespeichert, damit man es nicht immer wieder ein- 
geben muss und für andere Zwecke verwenden kann. Natürlich kann man es von Fall zu Fall modifizieren und den Gegebenheiten anpassen.

Interessant wird es, wenn man die gefällte Entscheidung, d. h. die gewählten Kandidaten, abspeichert. Häufig vergessen ja die Leute nach ein paar Monaten, wem sie alles die Stimme gegeben haben und verfolgen die von ihnen gewählten Kandidaten auch nicht bei ihrer Arbeit im Parlament. Auf MyPolitics lässt sich ein Monitoring der gewählten Kandidaten einrichten. Verhält sich ein Kandidat im Parlament nicht getreu seinen Wahlversprechungen, so wird dies erfasst. Wiederholt sich dies, so setzt man seinen Status auf ,Beobachtung' und erhält jedes Mal einen Alert, wenn dies erneut vorkommt. Natürlich kann man ihn/sie auch auf diese Diskrepanz hinweisen und einladen, sich zu rechtfertigen. Die so zusammenkommenden Informationen dienen dann bei den nächsten Wahlen als zusätzliche Entscheidungshilfen.

In Deutschland z. B. existiert die Plattform AbgeordnetenWatch.de, welche eine sachliche und individuelle Kommunikation zwischen Bürgerinnen und Bürgern sowie den Abgeordneten des Bundestages, der EU sowie unterschiedlicher Bundesländern zulässt. Alle Fragen und Antworten werden von einem Moderationsteam gegengelesen und nur aufgeschaltet, falls diese nicht gegen den publizierten Codex verstossen. Der Codex verbietet beispielsweise Fragen zum Privatleben sowie Beiträge, die Gewaltherrschaft, Rassismus, Sexismus oder politische und religiöse Verfolgung betreffen oder Massen-Mails.

Vorstellbar ist, über politische Plattformen wie eParticipation auch Initiativen und Referenden zu unterstützen und zu unterschreiben. Man kann sich beispielweise mit Gruppierungen, die ähnliche Werte vertreten, partnerschaftlich verlinken und wird jeweils bereits bei der Vorbereitung einer Initiative oder bei der Lancierung eines Referendums angefragt. So kann man Einfluss auf die Formulierung nehmen und Unterstützungsleistungen (Spenden, Weiterleitung von Information, usw.) anbieten.

Auf der Basis der politischen Profile können Gruppen von Gleichgesinnten (Political Communities of Interest) gebildet werden, die möglicherweise vom bestehenden Parteienangebot abweichen. Vernetzt kann man versuchen, Projekte zu entwickeln und auf die Politik Einfluss zu nehmen (Political Communities of Practice). Ist die Gruppe gross genug und formuliert konkrete Anliegen, so wird dies in den Medien aufgenommen oder allenfalls von Parteien und Volksvertretern ins politische Entscheidungssystem eingebracht. Denkbar ist, dass man sich direkt und elektronisch der Instrumente der direkten Demokratie bedient. Natürlich kann man sich virtuell mit Personen mit konträren Meinungen zum Gedankenaustausch treffen.

Von MyPolitics und OurPolitics ist es nur noch ein kleiner Schritt zum Public Memory, welches man sich als Verknüpfung der personenbasierten Webseiten in einer diachronen Perspektive vorstellen kann. Den Benutzern solcher Plattformen ist es freigestellt, ihre politischen Profile, Wahl- und Abstimmungsentscheidungen und Netzwerke anonymisiert öffentlich zu machen. Die so generierte Fülle an Daten gibt Auskunft über die Befindlichkeiten und Anliegen der Bürgerinnen und Bürger. Dies erfreut nicht nur die Forscher, welche neue Einblicke in die Gesellschaft und das politische Verhalten erhalten, sondern es ermöglicht auch, den sozialen Wandel und die veränderte Bedürfnisse viel besser und schneller zu erfassen und darauf einzugehen. 


\section{Paradigmenwechsel zur Demokratie 3.0}

Aus demokratietheoretischer Perspektive verkörpern MyPolitics und OurPolitics zwei unterschiedliche Erwartungen an das gute Funktionieren einer Demokratie. Für die Anhänger der liberalen Demokratietheorie handelt es sich bei der politischen Partizipation in erster Linie um einen individuellen Akt, bei dem Individuen ihre Interessen zum Ausdruck bringen. Wichtigste Ausdrucksform ist hier die Stimmabgabe an der Urne. Für die Anhänger der radikalen Demokratietheorien wird die politische Partizipation zu einem kollektiven und interaktiven Prozess, wie er beispielsweise in der Versammlungsdemokratie verwirklicht werden kann. In der Abb. 5 sind die wesentlichen Merkmale der Stimmabgabe für die Urnendemokratie wie für die Versammlungsdemokratie zusammengestellt.

Das eVoting unterstützt die Urnendemokratie, indem die Bürger orts- und zeitunabhängig ihre Stimme geheim abschicken können. Die vorgeschlagenen Optionen für MyPolitics wie freiwillige offene Stimmabgabe, Kommentare zur Stimmabgabe oder gar die Abgabe von Fuzzy Votes sorgt für fliessende Übergänge zwischen der Urnenund Versammlungsdemokratie. Umgekehrt können auf Partizipationsplattformen wie OurPolitics die Mitglieder sich bezüglich politischer Themen offen austauschen, vernetzen, gemeinsam politische Positionen erarbeiten und Initiativen lostreten. Mit unscharfen Matching-Verfahren erkennen sie Mitbürger oder Politiker, die dieselben politischen Ziele verfolgen. So können sie sich für ein politisches Anliegen vernetzen und gegenseitig stärken. Mit OurPolitics und radikaler Partizipation holen sie Teile direktdemokratischer Elemente in die digitale Gesellschaft zurück. Die Forderung der Netzgeneration, bei der Gestaltung der gesellschaftlichen Lebensbedingungen Mitwirkungs- und Entscheidungskompetenzen zu erlangen, wird weitgehend erfüllt. Zudem steht es den Nutzern von OurPolitics offen, von Fall zu Fall, wenn z. B. Persönlichkeitsrechte tangiert werden, geheime Abstimmungen durchzuführen. Dementsprechend bleiben für die digitale Gesellschaft auch hier fliessende Übergänge von der Versammlungsdemokratie OurPolitics hin zur Urnendemokratie MyPolitics.

Die hier ohne Zweifel positiv dargestellten Möglichkeiten der digitalen Partizipation mit MyPolitics resp. OurPolitics tönen verlockend. Community Building, grenzenlose Vernetzung, Gedankenaustausch mit Gleichgesinnten, Deliberation im herrschaftsfreien Raum, Entwicklung gemeinsamer Projekte, Mobilisierung und

Abb. 5 Liberale versus radikale Demokratiequalitäten angelehnt an (Schaub 2014, S. 30)
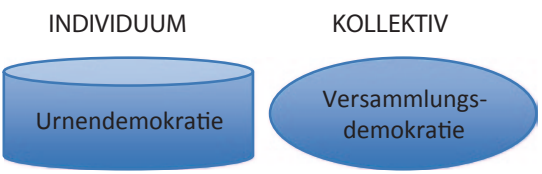

\begin{tabular}{l|l|l|}
\hline $\begin{array}{l}\text { Stimmabgabe } \\
\begin{array}{l}\text { Zeitliche Verteilung } \\
\text { der Stimmabgabe }\end{array}\end{array}$ & geheim & offen \\
\hline $\begin{array}{l}\text { Räumliche Verteilung } \\
\text { der Stimmabgabe }\end{array}$ & dezentral & zentral \\
\hline $\begin{array}{l}\text { Relation zwischen } \\
\text { Akteuren }\end{array}$ & Distanz & Kopräsenz \\
\hline
\end{tabular}


Partizipation, all dies könnten intelligente Internetapplikationen möglich machen. Und was will man mehr, als die gewählten Vertreter an den Schaltstellen der Macht zu kontrollieren und dafür zu sorgen, dass die Probleme und Anliegen des Volkes berücksichtigt werden. Damit kämen wir der Demokratie 3.0 (BITKOM 2013, Meisselbach 2009), der Synthese aus der attischen und der repräsentativen Demokratie, auch in einem grenzüberschreitenden Kontext, einen grossen Schritt näher.

Allerdings sollen die Risiken einer Entwicklung in Richtung digitaler Partizipation nicht ausgeblendet werden. Gross ist die Gefahr, dass nicht alle in gleichem Masse von den neuen Möglichkeiten Gebrauch machen können (Digital Divide). Können die Vernetzungsmöglichkeiten wirklich sinnvoll und demokratisch genutzt werden oder dienen sie vor allem sektenartigen Usergroups? Versinken wir nicht in einem Information Overload, oder werden die so erfassten Daten letztlich nicht zu Herrschaftswissen, welches die Grundgedanken einer demokratischen Gesellschaft verletzt?

Es bleibt zu hoffen, dass im Global Village in Zukunft Chancen und Risiken der digitalen politischen Partizipation in der Informations- und Wissensgesellschaft nicht zuletzt auch aus einer demokratietheoretischen Perspektive diskutiert, Nutzungsoptionen webbasierter Dienste gegeneinander abgewogen und Sicherheitslücken gestopft werden sowie Rechte zum Schutz der Privatsphäre aufrecht erhalten bleiben.

Danksagung Die Autoren bedanken sich bei Michael Mörike aus Deutschland sowie Jan Fivaz und Bruno Jeitziner aus der Schweiz für die kritischen Anmerkungen und Verbesserungsvorschläge zu einer Vorversion dieses Berichts.

\section{Literatur}

BITKOM (2013) BITKOM-Studie über Demokratie 3.0, Berlin, August 2013, http://www.bitkom.org/ files/documents/BITKOM-Studie_Demokratie_3.0_07-08-2013.pdf. Zugegriffen: 2. April 2014

Bundesrat (2002) Bericht des Bundesrates vom 9. Januar 2002 über den Vote électronique. Chancen, Risiken und Machbarkeit elektronischer Ausübung politischer Rechte

Corte-Real P (2007) Fuzzy voters, crisp votes. Int Game Theory Rev 9(1)67-86

Dalton RJ (1996) Comparative politics: micro-behavioural perspectives. In: Goodin RE, Klingemann HD (eds) A new handbook of political science. Oxford University Press, New York, S. 336-349

Dalton RJ, Wattenberg MP (1993) The not so simple act of voting. In: Finifter AW (ed) Political science. The state of the discipline II. The American Political Science Association, Washington, S. 193-218

De Rosa R (2010) cabina-elettorale.it. In: Cedroni L, Garzia D (eds) Voting advice applications in Europe. The state of the art. ScriptaWeb, Napoli, S. 187-198

Franklin MN, Mackie TT, Valen H (1992) Electoral change - Responses to evolving social and attitudinal structures in Western societies. Cambridge University Press, Cambridge

Jeitziner B, Meier A (2010) Optionen der Partizipation bei webbasierten Demokratieprozessen. In: Stember J, Eixelsberger W (Hrsg) Verwaltung im Wandel - Neue Anforderungen des modernen Verwaltungsmanagements in Mitteleuropa. LIT Verlag, Münster, S. 377-396

Ladner A (2009) Elektronisch wählen - smart wählen. In: Vatter A, Varone F, Sager F (Hrsg) Demokratie als Leidenschaft - Planung, Entscheidung und Vollzug in der schweizerischen Demokratie. Haupt, Bern, S. 205-224

Ladner A (2012) Voting Advice Applications werden im Wahlkampf immer wichtiger - Es ist Zeit, dass wir uns darüber Gedanken machen. In: Ziegler B, Wälti N (Hrsg) Wahl-Probleme der Demokratie. Schriften zur Demokratieforschung, herausgegeben durch das Zentrum für Demokratie Aarau. Schulthess-Verlag, Zürich, S. 91-110

Ladner A, Pianzola J (2010) Do voting advice applications have an effect on electoral participation and voter turnout? Evidence from the 2007 Swiss Federal Elections. In: Tambouris E, Macintosh A, Glassey O (eds) Electronic participation. Springer, Berlin, S. 211-224 
Ladner A, Schwarz D, Fivaz J (2008) Die Positionierung der Nationalratskandidierenden 2007. Eine explorative Analyse der Antworten auf die Fragen der Online-Wahlhilfe smartvote. Chavannes-prèsRenens: Cahier de l'IDHEAP Nr. 243

Ladner A, Felder G, Fivaz J (2010) More than toys? A first assessment of voting advice applications in Switzerland. In: Cedroni L, Garcia D (eds) Voting advice applications in Europe - The state of the art. Scriptaweb, Napoli, S. 91-123

Meier A (2009a) eDemocracy \& eGovernment - Entwicklungsstufen einer demokratischen Wissensgesellschaft. Springer, Berlin

Meier A (2009b) Elektronische Abstimmungen und Wahlen. In: Hofmann J, Reich S (Hrsg) eGovernment. HMD-Heft Nr. 265, dpunkt Verlag, Heidelberg, S. 51-61

Meier A, Teran L (2012) Wahlhilfesystem für elektronische Wahlen unter Nutzung der unscharfen Logik. In: Eixelsberger W, Stember J (Hrsg) E-Government - Zwischen Partizipation und Kooperation. Springer, Wien, S. 73-87

Meisselbach C (2009) Web 2.0- Democracy 3.0? Demokratische Potentiale des Internets. Nomos, Baden-Baden

Schaub H-P (2014) Landsgemeinde oder Urne - was ist demokratischer? Ein Vergleich der demokratischen Qualitäten von Urnen- und Versammlungsdemokratien in den Schweizer Kantonen. Dissertation Universität Bern

Serres M (2013) Erfindet euch neu! Eine Liebeserklärung an die vernetzte Generation. Suhrkamp Verlag, Berlin

Teran L (2014) SmartParticipation - A fuzzy-based recommender system for political community-building. Springer, Berlin

Zadeh LA (1965) Fuzzy Sets. Information and Control 8(3)338-353 